\title{
Triplet Superconductivity in a Nutshell
}

\author{
Kazumi Maki · Hae Young Kee · Yoshifumi Morita
}

Received: 10 October 2008 / Accepted: 13 October 2008 / Published online: 29 October 2008

(C) The Author(s) 2008. This article is published with open access at Springerlink.com

\begin{abstract}
The triplet superconductors have been around us since 1980, when Jerome et al. discovered the Bechgaard salts (TMTSF) ${ }_{2} \mathrm{PF}_{6}$ and (TMTSF) ${ }_{2} \mathrm{ClO}_{4}$. Now there are more than 20 or so triplet superconductors discovered. Recently we found that most of them with the known gap symmetries can be mapped to the superfluid phase of ${ }^{3} \mathrm{He}-\mathrm{A}$ and ${ }^{3} \mathrm{He}-\mathrm{A}_{1}$. Further, in most of them, $\hat{l}$ (the chiral vector, i.e. the quantization axis of the pair angular momentum) is fixed parallel to one of the crystal axes and all the topological defects are considered in terms of $\hat{d}$-textures, where $\hat{d}$ is the spin vector. Then $\mathrm{Sr}_{2} \mathrm{RuO}_{4}, \mathrm{UPt}_{3}, \mathrm{PrOs}_{4} \mathrm{Sb}_{12}$ and (TMTSF) ${ }_{2} \mathrm{ClO}_{4}$ belong to type-A, which is an analog of superfluid ${ }^{3} \mathrm{He}-\mathrm{A}$. In these superconductors, a vortex splits into a pair of half quantum vortices (HQVs) at low temperatures. On the other hand, $\mathrm{CePt}_{3} \mathrm{Si}, \mathrm{CeIrSi}_{3}, \mathrm{CeRhSi}_{3}$, $\mathrm{UIr}$ and $\mathrm{Li}_{2} \mathrm{Pt}_{3} \mathrm{~B}$ (those in non-centrosymmetric crystals) belong to type- $\mathrm{A}_{1}$, which is an analog of superfluid ${ }^{3} \mathrm{He}-\mathrm{A}_{1}$. In all of these triplet superconductors, vortices harbor the zero mode or the Majorana fermions, the implications of which deserves further exploration.
\end{abstract}

\section{K. Maki}

Max-Planck Institute for the Physics of Complex Systems,

Nöthnitzer Str. 3801187 Dresden, Germany

\section{K. Maki}

Department of Physics and Astronomy, University of Southern California, Los Angeles, CA 90089-0484, USA

H.Y. Kee

Department of Physics, University of Toronto, Toronto,

Ontario M5S 1A7, Canada

\section{Y. Morita $(\bowtie)$}

Faculty of Engineering, Gunma University, Kiryu,

Gunma 376-8515, Japan

e-mail: morita@phys.sci.gunma-u.ac.jp
Keywords Unconventional superconductivity $\cdot$ Half quantum vortex

\section{Introduction}

The first triplet superconductors have been discovered in 1980 by Jerome et al. [1] in Bechgaard salts (TMTSF) ${ }_{2} \mathrm{PF}_{6}$ and (TMTSF) ${ }_{2} \mathrm{ClO}_{4}[2,3]$. Since then many triplet superconductors have been discovered, which is concisely reviewed by Sigrist and Ueda [4]. Although it has been noticed that all of these triplet superconductors are nodal (i.e. non s-wave), the gap symmetry has not been addressed seriously until around 1994. We now have a proposal on the gap symmetry of $\mathrm{UPt}_{3}, \mathrm{Sr}_{2} \mathrm{RuO}_{4}$ and $\mathrm{PrOs}_{4} \mathrm{Sb}_{12}$ as shown in Fig. 1 $[5,6]$. Here we understand that these triplet superconductors are characterized by the equal spin pairing (ESP) and with extremely small spin-orbit coupling energy $E_{s o} \sim 10^{-3} \Delta$, where $\Delta$ is the superconducting energy gap [7]. Therefore the superconducting order parameter is characterized by $\hat{l}$ (the chiral vector) and $\hat{d}$ (the spin vector) as in superfluid ${ }^{3} \mathrm{He}-\mathrm{A}$, except for the gap symmetry $[8,9]$.

On the other hand, the discovery of a non-centrosymmetric superconductor $\mathrm{CePt}_{3} \mathrm{Si}$ by Bauer et al. in 2004 [10] opened a new door. According to Anderson's argument [11], such a triplet superconductor cannot exist. In order to clarify the role of the parity-breaking term (e.g. the Rashba term [12]), Frigeri et al. [13, 14] considered a model with a Rashba term. They found, first of all, the Fermi surface is split into the one for up-spin and the another for downspin, as shown in Fig. 2. Here we have introduced the Rashba Hamiltonian $\mathcal{H}_{R}=\alpha k_{z} \sigma_{z}$, different from the one used by Frigeri et al. $[13,14]$. Secondly, they found the strong admixture of the singlet component. Later, many noncentrosymmetric superconductors have been discovered in 


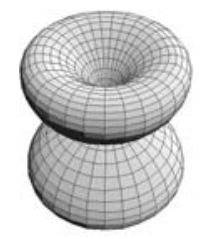

$Y_{3, \pm 2}(\theta, \varphi)$

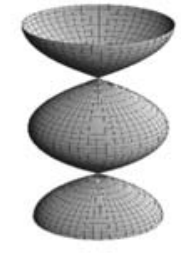

chiral f-wave

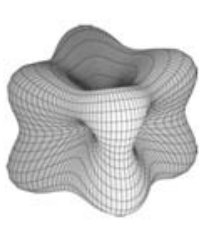

$(\mathrm{p}+\mathrm{h})$-wave
Fig. 1 Our proposal on gap functions of $Y_{3, \pm 2}(\theta, \varphi)$ for $\mathrm{UPt}_{3}$ and $\mathrm{CePt}_{3} \mathrm{Si}$, chiral $\mathrm{f}$-wave for $\mathrm{Sr}_{2} \mathrm{RuO}_{4},(\mathrm{p}+\mathrm{h})$-wave for $\mathrm{PrOs}_{4} \mathrm{Sb}_{12}$

Fig. 2 The split Fermi surface for each spin due to the parity-breaking term

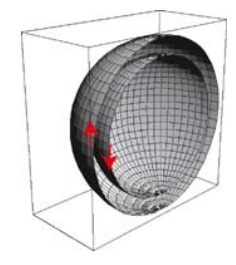

$\mathrm{CeIrSi}{ }_{3}, \mathrm{CeRhSi}_{3}, \mathrm{UIr}$ and $\mathrm{Li}_{2} \mathrm{Pt}_{3} \mathrm{~B}$ [15-18]. Also the spinorbit coupling energy $E_{s o}$ or the Rashba term is found invariably extremely large $[15,18]$, i.e. $E_{s o} \sim 10^{3} \mathrm{~K}$ in these systems. Recently in [19], we have proposed that the triplet superconductors in non-centrosymmetric crystals should be like the one in ${ }^{3} \mathrm{He}-\mathrm{A}_{1}$, in contrast to the previous proposals. The superconductivity occupies the Fermi surface associated with one spin component (say, up-spin) while the other Fermi surface remains in the normal state. With this assumption, we can describe many unusual experimental results of $\mathrm{CePt}_{3} \mathrm{Si}$.

\section{The Zero Mode and the Majorana Fermion}

In this section, we shall summarize the bound-state spectrum around an Abrikosov's vortex in triplet superconductors. In both type-A and type- $\mathrm{A}_{1}$ triplet superconductors, we conclude that vortices harbor the zero mode which behaves as the Majorana fermion.

First of all, we note that $\hat{l}$ (the chiral vector) of many triplet superconductors are fixed parallel to the crystal $\hat{c}$-axis. Secondly, in the absence of a magnetic field, $\hat{d} \| \hat{l}$ is due to the spin-orbit coupling. However, when the magnetic field $\vec{H}$ (parallel to $\hat{l}$ ) is applied in the vicinity of $H_{c 2}(T)$ (the upper critical field), $\hat{d} \perp \hat{l}$ is realized. In this particular situation, Ivanov $[20,21]$ have shown that the BdG equations for the triplet superconductor decouple into the one for the up-spin component and the other for the down-spin component. Then these BdG equation have the same structure as the one for s-wave superconductor first written down in $[22,23]$. Therefore, first let us consider a single vortex.

Following Caroli, de Gennes and Matricon [22, 23], the bound state energy formula is given as follows (see, for detail [24-26])

$\epsilon_{n}=n \omega_{0} \quad$ with $n=0, \pm 1, \pm 2, \pm 3, \ldots$, where $\omega_{0} \simeq \Delta\left(p_{F} \xi\right)^{-1}\left[\ln \left(\frac{2}{\delta}\right)\right]^{-1}$ ( $\delta$ is the cut-off parameter) and $\delta \simeq \sqrt{\Gamma / \Delta}$ ( $\Gamma$ is the quasiparticle scattering rate in the normal state) in the very clean system. So $\omega_{0}$ is essentially the same as in s-wave superconductors except for the logcorrection due to the nodal structure. Note that the boundstate spectrum is very different from the one for the singlet superconductors (s-wave, d-wave etc.). For the singlet superconductors, $\epsilon_{n}=\left(n+\frac{1}{2}\right) \omega_{0}$ with $n=0, \pm 1, \pm 2, \pm 3, \ldots$ with a gap $\omega_{0} / 2 \sim \Delta^{2} / E_{F}$. On the other hand, in triplet superconductors, there is the zero mode or the Majorana fermion $[9,27,28]$ with zero energy (right on the Fermi surface). The Majorana fermion wavefunction associated with the zero mode is given by

$\phi_{0}(r, \hat{k}) \sim[\operatorname{sech}(r / \xi)]^{C|f|}$

and $C=\Delta \xi v^{-1} \sim 1$. These wavefunctions should be accessible at low temperatures.

\section{Half-Quantum Vortices}

Half-quantum vortices are generated in type-A triplet superconductors, which correspond to the $\hat{d}$-soliton or the $\hat{d}$-domain wall [24-26]. Since $\hat{l}$-vector is fixed parallel to the crystalline $\hat{c}$-axis, we limit ourselves to the texture free energy associated with the $\hat{d}$ vector. Especially for $\mathrm{UPt}_{3}$, $\mathrm{Sr}_{2} \mathrm{RuO}_{4}$ and $\mathrm{PrOs}_{4} \mathrm{Sb}_{12}$ in a magnetic field parallel to $\hat{l}$, the texture free energy simplifies as [29]

$$
\begin{aligned}
\mathcal{F}= & \frac{1}{2} \chi_{N} C^{2} \int d x d y\left[K(\nabla \Phi)^{2}+\sum_{i, j}\left|\partial_{i} \hat{d}_{j}\right|^{2}\right. \\
& \left.+\xi_{D}^{1-2}\left(\hat{d}_{x}^{2}+\hat{d}_{y}^{2}\right)\right]
\end{aligned}
$$

where $\chi_{N}$ and $C$ are the spin susceptibility and the spinwave velocity, and $\xi_{D}^{1-2}=\xi_{D}^{-2}-\xi_{H}^{-2}$. Here

$$
K(t)=\frac{\rho_{s}(t)}{\rho_{s p}(t)}=\frac{1+\frac{F_{1}}{3}}{1+\frac{F_{1}^{a}}{3}} \frac{1+\frac{F_{1}^{a}}{3}\left(1-\rho_{s}^{0}(t)\right)}{1+\frac{F_{1}}{3}\left(1-\rho_{s}^{0}(t)\right)},
$$

where $t=T / T_{c}, \rho_{s}(t), \rho_{s p}(t)$ and $\rho_{s}^{0}(t)$ are the superfluid density, the spin superfluid density and the bare superfluid density. In $\mathrm{PrOs}_{4} \mathrm{Sb}_{12}, \rho_{s}^{0}(t)$ is well approximated by $\rho_{s}^{0}(t)=1-0.460572 t-0.53428 t^{1.729}$ [30]. Experimentally, $m^{*} / m=50$ in $\mathrm{PrOs}_{4} \mathrm{Sb}_{12}$ and, if we ignore $F_{1}^{a}$,

$K(t)=\left[1-\left(1-\frac{m}{m^{*}}\right) \rho_{s}^{0}(t)\right]^{-1}$.

Here we note that the temperature dependence of $K(t)$ in $\mathrm{UPt}_{3}$ and $\mathrm{Sr}_{2} \mathrm{RuO}_{4}$ is very similar to that of $\mathrm{PrOs}_{4} \mathrm{Sb}_{12}$ $[24,25]$. 
Here we shall compare the free energy of an Abrikosov's vortex and a bound pair of half-quantum vortices by the $\hat{d}$-soliton. For this purpose, we consider the free energy of an Abrikosov's vortex in a unit circular cell with radius $a$ which encloses a flux quantum. Then $a$ is given by

$a=\left(\frac{\phi_{0}}{\pi H}\right)^{1 / 2}$,

where $H$ is the field strength. Note also a similar relation due to de Gennes:

$H_{c 2}(t)=\frac{\phi_{0}}{2 \pi \xi^{2}(t)}$,

where $\xi(t)$ is the coherence distance. This indicates, for $H<H_{c 2}(t), a>\sqrt{2} \xi(t)$. The free energy for an Abrikosov's vortices is readily given by

$\mathcal{F}_{A}=\pi \chi_{N} C^{2} K \ln (a / \xi)$.

For a bound pair of HQVs separated by a distance $R$ and inside the unit cell,

$$
\begin{aligned}
\mathcal{F}_{B P}= & \frac{\pi}{2} \chi_{N} C^{2}\left\{K \ln \left(\frac{a+\sqrt{a^{2}-R^{2} / 4}}{2 \xi}\right)+\ln (a / \xi)\right. \\
& -\frac{R}{4 a} \sin ^{-1}\left(\frac{R}{\sqrt{a^{2}-R^{2} / 4}}\right) \\
& \left.+\frac{1}{4}\left(\frac{R}{\xi_{D}^{\prime}}\right)^{2} \ln \left(\frac{2 \xi_{D}^{\prime}}{a} \frac{K+1}{\sqrt{2 K+1}}\right)\right\} .
\end{aligned}
$$

Also, for $K(t) \gg 1$, this equation is optimized at $R / 2 a=$ $\sqrt{2 K+1} / K+1$ and [31]

$$
\begin{aligned}
\mathcal{F}_{B P}= & \frac{\pi}{2} \chi_{N} C^{2}\left\{K \ln \left(\frac{a}{2 \xi} \frac{2 K+1}{K+1}\right)+\ln \left(\frac{a}{\xi}\right)\right. \\
& -\frac{1}{2} \frac{\sqrt{2 K+1}}{K+1} \sin ^{-1}\left(\frac{2 \sqrt{2 K+1}}{K+1}\right) \\
& \left.+\frac{2 K+1}{(K+1)^{2}}\left(\frac{a}{\xi_{D}^{\prime}}\right)^{2} \ln \left(\frac{2 \xi_{D}^{\prime}}{a} \frac{K+1}{\sqrt{2 K+1}}\right)\right\} .
\end{aligned}
$$

Now from $\mathcal{F}_{A}=\mathcal{F}_{B P}$, we find that

$$
\begin{aligned}
H^{*} & (t) / H_{c 2}(t) \\
& =\left[2^{-(K-2)}(2 K+1)^{(2 K+1)}(K+1)^{-2(K+1)}\right]^{-1 /(K-1)},
\end{aligned}
$$

where $H^{*}(t)$ is the phase boundary below which HQVs are stable. For $\mathrm{PrOs}_{4} \mathrm{Sb}_{12}$, we show the stability region of HQVs in Fig. 3 [26], which is close to the phase boundary determined experimentally by Izawa et al. [32]. According to the our theoretical picture, the B phase discovered in [32] is

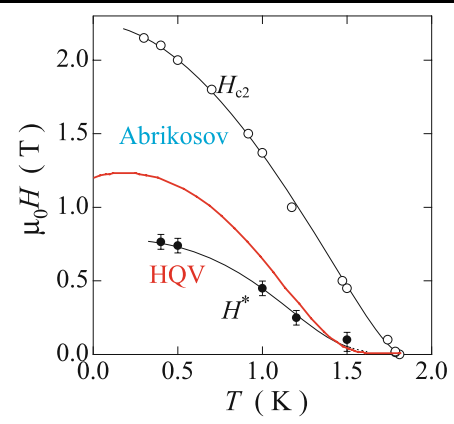

Fig. 3 For $\mathrm{PrOs}_{4} \mathrm{Sb}_{12}$, the stability region of HQV (inside the line without circles) is shown, based on our theoretical result [26]. It is close to the phase (whose boundary is given by black circles) determined experimentally by Izawa et al. [32]

the vortex state filled with HQVs. Also we have got a similar result for the $\mathrm{B}$ phase in $\mathrm{UPt}_{3}[24,25]$. On the other hand, as to $\mathrm{Sr}_{2} \mathrm{RuO}_{4}$, there is no clear hallmark of such a phase transition. However, we speculate that the anomalous angle-dependent magneto-specific heat found by Deguchi et al. [33] below $300 \mathrm{mK}$ can be due to HQVs.

Finally, it is to be noted that we can access the HQVs by scanning tunneling microscopy (STM) [34], micromagnetometry [35] and small angle neutron scattering (SANS) at ultra-low temperature.

\section{Concluding Remarks}

We have proposed a new paradigm for triplet superconductivity.

(1) Most of triplet superconductors, which were discovered before 2004, belong to type-A. Their topological structure is very similar to superfluid ${ }^{3} \mathrm{He}-\mathrm{A}$. At low temperatures, a vortex splits into a pair of half-quantum vortices (HQVs).

(2) A new class of superconductors in non-centrosymmetric crystals, which were discovered after 2004, belong to type- $A_{1}$. The topological structure is the same as of superfluid ${ }^{3} \mathrm{He}-\mathrm{A}_{1}$.

(3) All these triplet superconductors have the zero mode/ Majorana fermion attached to a vortex, the implications of which should be explored further.

Acknowledgements We thank Ana Celia Mota and Coneliu Florian Miclea for very informative discussions on the ongoing experiments on the flux pinning in $\mathrm{PrOs}_{4} \mathrm{Sb}_{12}$ and $\mathrm{CePt}_{3} \mathrm{Si}$. We thank also Balazs Dora, Stephan Haas and Masaru Kato for enlightening discussions. Y.M. is supported by a Grant-in-Aid for Scientific Research from the Ministry of Education, Culture, Sports, Science and Technology of Japan.

Open Access This article is distributed under the terms of the Creative Commons Attribution Noncommercial License which permits any noncommercial use, distribution, and reproduction in any medium, provided the original author(s) and source are credited. 


\section{References}

1. Jerome, D., Mazaud, A., Ribault, M., Bechgaard, K.: J. Phys. Lett. 41, L95 (1980)

2. Takigawa, M., Yasuoka, H., Saito, G.: J. Phys. Soc. Jpn. 56, 873 (1987)

3. Lee, I.J., Brown, S.E., Naughton, M.J.: J. Phys. Soc. Jpn. 75, 051011 (2006)

4. Sigrist, M., Ueda, K.: Rev. Mod. Phys. 63, 239 (1991)

5. Won, H., Haas, S., Parker, D., Telang, S., Vanyolos, A., Maki, K.: In: Lectures on the Physics of Highly Correlated Electron Systems IX. AIP Conference Proceedings, vol. 789. Melville, Brooklyn (2005)

6. Won, H., Morita, Y., Maki, K.: Phys. Status Solidi B 244, 4371 (2007)

7. Maki, K., Haas, S., Parker, D., Won, H.: In: Topology in Condensed Phases. World Scientific, Singapore (2006)

8. Vollhardt, D., Wölfle, P.: The Superfluid Phases in Helium Three. Taylor \& Francis, London (1990)

9. Volovik, G.E.: The Universe in a Helium Droplet. Clarendon, Oxford (2003)

10. Bauer, E., Hilscher, G., Michor, H., Paul, C., Scheidt, E.W., Gribanov, A., Seropegin, Yu., Noël, H., Sigrist, M., Rogl, P.: Phys. Rev. Lett. 92, 027003 (2004)

11. Anderson, P.W.: Phys. Rev. B 30, 4000 (1994)

12. Rashba, E.J.: Sov. Phys. Solid State 2, 1109 (1960)

13. Frigeri, P.A., Agterberg, D.F., Koga, A., Sigrist, M.: Phys. Rev. Lett. 92, 097001 (2004)

14. Frigeri, P.A., Agterberg, D.F., Sigrist, M.: New J. Phys. 6, 115 (2004)

15. Settai, R., Takeuchi, T., Onuki, Y.: J. Phys. Soc. Jpn. 76, 051003 (2007)
16. Kimura, N., Muro, Y., Aoki, H.: J. Phys. Soc. Jpn. 76, 051010 (2007)

17. Kobayashi, T.C., Hori, A., Fukushima, S., Hidaka, H., Kotegawa, H., Akazawa, T., Takeda, K., Ohishi, Y., Yamamoto, E.: J. Phys. Soc. Jpn. 76, 051007 (2007)

18. Nishiyama, M., Inada, Y., Zheng, G.-Q.: Phys. Rev. Lett. 98, 047002 (2007)

19. Morita, Y., Maki, K.: Preprint (2008)

20. Ivanov, D.A.: In: Vortices in Unconventional Superconductors and Superfluids. Springer, Berlin (2002)

21. Ivanov, D.A.: Phys. Rev. Lett. 40, 268 (2001)

22. Caroli, C., de Gennes, P.G., Matricon, J.: Phys. Lett. 9, 310 (1964)

23. de Gennes, P.G.: The Superconductivity of Metals and Alloys. Benjamin, New York (1966). Reprinted in Percus Book, Reading (1999)

24. Kee, H.Y., Maki, K.: Preprint (2008)

25. Kee, H.Y., Raghavan, A., Maki, K.: Preprint (2007)

26. Morita, Y., Maki, K.: Preprint (2008)

27. Majorana, E.: Nuovo Cim. 14, 171 (1937)

28. Penrose, R.: The Road to Reality. Vintage Books, New York (2007)

29. Kee, H.Y., Kim, Y.B., Maki, K.: Phys. Rev. B 62, R9275 (2000)

30. Parker, D., Maki, K., Haas, S.: Eur. Phys. J B 49, 77 (2006)

31. Kee, H.Y., Maki, K.: Europhys. Lett. 80, 46003 (2007)

32. Izawa, K., Nakajima, Y., Goryo, J., Matsuda, Y., Onuki, S., Settai, R., Thalmeier, P., Maki, K.: Phys. Rev. Lett. 90, 117001 (2003)

33. Deguchi, K., Mao, Z.Q., Maeno, Y.: J. Phys. Soc. Jpn. 73, 1313 (2004)

34. Fischer, Ø., Kugler, M., Maggio-Aprile, I., Berthod, C., Renner, C.: Rev. Mod. Phys. 79, 353 (2007)

35. Tsuei, C.C., Kirtley, J.R.: Rev. Mod. Phys. 72, 969 (2000) 